Research Article

\title{
Evolutionary Game Analysis of Promoting Industrial Internet Platforms to Empower Manufacturing SMEs through Value Cocreation Cooperation
}

\author{
Dongdong Li $(D)$, Chunfa Li $(D)$, and Runde Gu $(\mathbb{D}$ \\ School of Management, Tianjin University of Technology, Tianjin 300384, China \\ Correspondence should be addressed to Chunfa Li; lcf@tjut.edu.cn
}

Received 26 July 2021; Revised 18 August 2021; Accepted 1 September 2021; Published 11 September 2021

Academic Editor: Daqing Gong

Copyright ( 2021 Dongdong Li et al. This is an open access article distributed under the Creative Commons Attribution License, which permits unrestricted use, distribution, and reproduction in any medium, provided the original work is properly cited.

\begin{abstract}
A good partnership is conducive to promoting the empowerment of manufacturing small- and medium-sized enterprises (SMEs) via industrial Internet platforms. By analyzing the factors influencing the cooperation motives of both parties and individual behavior, this paper puts forward the design of a cost-sharing and scale revenue-sharing mechanism and establishes an evolutionary game model. Then, the evolutionary stability strategies (ESSs) of individuals and the evolutionary equilibrium state of the system are analyzed. The results show that the key factors affecting the strategic choices of industrial Internet platforms and manufacturing SMEs are different and will change with the number of platform customers and the level of digitalization of enterprises. By sharing the access cost of SMEs and the scale revenue of the platform, mutual trust between the two parties can be enhanced, and SMEs will be more motivated to access the platform. Moreover, the platform network externality, customer churn risk, and cost-sharing ratio have different influences on the process of reaching evolutionary equilibrium in the system. Collaborative revenue expectations are critical to the behavioral strategies of both parties. In comprehensive consideration of the results of this study, it is recommended that industrial Internet platforms be subsidized in the initial stage of cooperation.
\end{abstract}

\section{Introduction}

The deep integration of new-generation information technology and the manufacturing industry has reshaped the production organization mode of the traditional manufacturing industry and promoted the formation of new modes with intelligence as the core feature [1]. Industrial Internet platforms, which represent comprehensive information infrastructure for developing industrial intelligence, are regarded as the key support of the fourth industrial revolution and the future direction of industrial development $[2,3]$. Since General Electric first proposed the concept of the industrial Internet in 2012 [4], the major manufacturing countries have successively established industrial Internet industry alliances and have formulated strategic plans for the development of advanced manufacturing industries including the industrial Internet $[5,6]$. Later, with the improvement of the industrial Internet architecture, industrial Internet platforms with different core businesses, such as Predix, MindSphere, INDICS, and Root Cloud, have emerged [7, 8]. An industrial Internet platform improves the entire value chain from research and development $(\mathrm{R} \& \mathrm{D})$ to the market by connecting massive industrial equipment and collecting modeling data for industrial institutions. Based on the value mining of massive industrial data, intelligent production decision-making becomes possible, which has had a profound impact on the traditional manufacturing industry. Due to the substantial application and enabling value of industrial Internet platforms, they have become a new focus of industrial competition in various countries [9].

Two core features of industrial Internet platforms are their intelligent application and data-driven characteristics [10], which place higher requirements on the level of digitalization equipment and the production organization of manufacturing enterprises. The enabling function of 
industrial Internet platforms is closely related to the level of digitalization of manufacturing enterprises [11]. However, it is difficult and costly to improve the digitalization of manufacturing enterprises, especially for small- and medium-sized enterprises (SMEs) with weak comprehensive strength. Due to the high heterogeneity of equipment in different industries and the difficulties in real-time data collection and modeling, it is difficult to give full play to the traffic advantages of industrial Internet platforms in a short period of time [12]. Therefore, it is necessary to establish a closer cooperation and trust relationship between industrial Internet platforms and manufacturing enterprises $[13,14]$.

In addition to the difficulties caused by a lack of professional knowledge and comprehensive strength, the motivation to protect trade secrets and concerns about security risks also weaken the willingness of manufacturing SMEs to access industrial Internet platforms [15]. However, at present, the mainstream industrial Internet platforms are more aimed at technical services and ignore the willingness of SMEs to access the platform and their difficulties in doing so [16]. Via the construction of an effective cooperation mode, not only can platforms be promoted to give full play to their enabling role, but platforms and enterprises can also be assisted to achieve value cocreation [17]. Therefore, determining how to innovate the cooperation mechanism between industrial Internet platforms and manufacturing SMEs and achieve win-win cooperation is particularly critical.

Due to the inherent interdependence between industrial Internet platforms and manufacturing SMEs, individuals are no longer simply pursuing the maximization of their interests. Because of the contradiction between individual behavioral strategies and overall interests, it is necessary to design a reasonable mechanism to guarantee the ultimate goal of the system. On the premise of individual bounded rationality, an evolutionary game can effectively reflect the interaction between individuals in the micro-state and changes in the population structure at the meso-level [18]. Therefore, an evolutionary game can provide in-depth practical insights into the empowerment of SMEs by industrial Internet platforms. This paper focuses on the following research questions: (1) How can the cooperation between an industrial Internet platform and manufacturing SMEs be promoted via effective mechanical design? (2) What is the interactive mechanism of the behavior strategies of both parties under the influence of bounded rationality? (3) How do the main related factors affect individual behavioral strategies and the equilibrium state of system evolution?

Motivated by these research questions, this work analyzes the motivation of cooperation between industrial Internet platforms and manufacturing SMEs and designs a cost-sharing and scale revenue-sharing mechanism to promote cooperation. Considering the factors influencing individual behavior, an evolutionary game model is constructed to analyze the interaction mechanism of the strategies of both parties. Then, the evolutionary stability strategies (ESSs) of individuals and the equilibrium state of the system are explored based on the evolutionary game analysis. Finally, the effects of key factors on the evolution of the equilibrium state of the system under different constraint scenarios are discussed based on simulations.

The remainder of this paper is organized as follows. Section 2 presents a review of related literature, and Section 3 describes the fundamental assumption and model settings. Section 4 analyzes the evolution path and stability of the strategies of industrial Internet platforms and manufacturing SMEs. Section 5 discusses the strategy of evolution stability under the interaction of the two parties. Section 6 simulates the influences of different main factors on the evolution of the system to the equilibrium state. Finally, this work is concluded in Section 7.

\section{Literature Review}

This section reviews related literature spanning two research streams, namely, industrial Internet platforms and the transformation and upgrading of manufacturing enterprises.

2.1. Industrial Internet Platforms. As key supporting facilities for the development of industrial intelligence, the core value of industrial Internet platforms lies in providing application services to enterprises [19]. Industrial Internet platforms can provide applications (APPs) and customized services for enterprises via the calculation of massive data, application development, and knowledge encapsulation, which can effectively reduce the access threshold of manufacturing SMEs [20]. With the continuous improvement of the architecture and functions of industrial Internet platforms, their application in different industries has attracted widespread attention [21].

Li et al. [22] systematically classified the changes brought by industrial Internet platforms to the fields of energy, healthcare, manufacturing, smart factories, etc. Arnold et al. [23] studied the impact of the industrial Internet on business models of different industrial sectors via exploratory multicase research methods. Kiel et al. [24] found that the emergence of the industrial Internet has had a huge impact on the established business models of manufacturing companies. Shan et al. [8] reviewed the development of Industry 4.0 and analyzed Sany's application of the industrial Internet and the process of achieving smart manufacturing transformation. In addition, due to the critical role of privacy and security in industrial production, the security protection and risk prevention issues of industrial Internet platforms have also become the focus of research [25].

In general, the current research on industrial Internet platforms has focused on three areas, namely, their architecture [26], key technologies [19], and industrial applications [27]. However, few studies have focused on the dilemma of access to industrial Internet platforms from the perspective of enterprises. In essence, as an enabling service tool, the ultimate goal of industrial Internet platforms is to provide support services for industrial production. At present, research on industrial Internet platforms is mainly focused on the technical level, whereas fewer investigations have been conducted from the management level [28]. Thus, 
the ability of the services of industrial Internet platforms to better meet the needs of enterprises must be strengthened, which would be beneficial to the development of both platforms and enterprises [29].

\subsection{Transformation and Upgrading of Manufacturing} Enterprises. The emergence of industrial Internet platforms has had a profound impact on the production organization and business operation mode of traditional manufacturing enterprises [30]. Manufacturing enterprises are facing increasingly serious challenges in production organization, resource scheduling, and business models. With the rapid development of information technology and changes in market demand, the digital and intelligent transformation of the traditional manufacturing industry has become inevitable [31].

The digital transformation of manufacturing enterprises is a complicated process that requires significant resources and capital investment [32]. SMEs often face more difficulties than large enterprises in the process of transformation and upgrading due to technological disadvantages and insufficient funds [33]. Mittal et al. [34] discussed the needs of SMEs in terms of capital, R\&D capabilities, and expertise in the Industry 4.0 era. Digital transformation is a disruptive change in industry, and industrial Internet platforms offer great potential for SMEs to transform and upgrade [35]. Hansen and Bøgh [36] found that the application rates of artificial intelligence and the Internet of Things in SMEs remain low. However, against the background of intelligent manufacturing, it is particularly important for the overall upgrading of the industry for manufacturing SMEs with different professional strengths to access the intelligent manufacturing network [37]. However, compared to large enterprises, SMEs have many difficulties in terms of technology uptake, expertise, and financial support, which also leads to a lack of capacity and motivation to access industrial Internet platforms [16, 38]. Thus, determining how to overcome these constraints and realize intelligent development with the help of industrial Internet platforms has become an urgent problem for manufacturing SMEs and would also be beneficial to the continuous upgrading of the industrial Internet platform.

Most of the previous studies on industrial Internet platforms have focused on the technical perspective. While a few studies have focused on their application in different industries and their impact on enterprises, they have been limited to the superficial analysis of the current application state or the discussion of individual cases. Overall, there has been a lack of research on the willingness of enterprises to access industrial Internet platforms and the related difficulties. However, to some extent, consumer demand and market adoption are the keys to the success of emerging technologies [39]. Regarding SMEs as the main manufacturing industry, their access to industrial Internet platforms is particularly important to realize comprehensive intelligent manufacturing. There currently exists a mismatch between the service supply of industrial Internet platforms and the real needs of manufacturing enterprises, and little research has focused on this contradiction. The solution to this issue will help enhance the enabling effect of industrial Internet platforms on manufacturing enterprises, which will ultimately promote the realization of intelligent manufacturing.

\section{Problem Description and Modeling}

In view of the complexity of industrial Internet application scenarios and the diversity of the needs of manufacturing enterprises, a stable value cocreation relationship and an orderly self-growth mechanism must be established between the two parties. Similar to the e-commerce platforms, industrial Internet platforms accumulate competitive advantages by expanding their customer scale in the early stage of development. However, the cost of accessing industrial Internet platforms is huge, and the industry knowledge barriers and business data risk also hinder the enthusiasm of manufacturing SMEs.

In view of this dilemma, industrial Internet platforms can consider sharing the platform access costs and scale benefits with enterprises. The sharing of access costs includes providing technical guidance, professional consultation, or price concessions for enterprises to access the platform; this can reduce the burden of manufacturing SMEs while also enhancing the attractiveness of the platform. Scale revenue mainly refers to the advantages of data volume and market competition brought by the enterprise accessing the platform. An incremental gain in scale can reflect the marginal contributions of different companies with access to the platform to the competitiveness of the platform. Access cost-sharing and scale benefit-sharing can reduce the entry barrier of the platform and enhance the trust of enterprises, which essentially reflects a value cocreation behavior with customer enterprises led by the platform. This will also help the platform rapidly expand its customer scale and gain competitive advantage in the market.

The present study focuses on the choices of cooperation strategies by an industrial Internet platform and manufacturing SMEs. The industrial Internet platform adopts a value cocreation strategy, i.e., sharing access costs and scale revenue with manufacturing enterprises, which is convenient for the platform to quickly gain scale advantages. However, this will result in increased expenses for the platform and a loss of scale revenue. The resulting changes in revenue are uncertain, which adds complexity to the platform's choice of strategy. The access of manufacturing SMEs to the industrial Internet platform can help improve their production and operation efficiency, expand their business scope, and enhance their ability to cope with risks. However, due to the large initial investment, the high demand for industry knowledge, and the high risk of trade secret disclosure, SMEs are faced with a dilemma when choosing access strategies. The strategic choices of industrial Internet platforms and SMEs interact with each other, and, under the influence of bounded rationality, the strategic choices of both parties are an evolutionary game process, which is subsequently modeled. 
3.1. Model Hypothesis and Payment Matrix. Let the strategic space of the industrial Internet platform $(P)$ be $S_{P}=\left\{S_{P}^{1}, S_{P}^{2}\right\}$, and let the strategic space of manufacturing SMEs $(M)$ be $S_{M}=\left\{S_{M}^{1}, S_{M}^{2}\right\}$. Among them, $S_{P}^{1}$ and $S_{P}^{2}$, respectively, represent the strategies of adopting and not adopting value cocreation, and $S_{M}^{1}$ and $S_{M}^{2}$, respectively, represent the strategies of accessing and not accessing the platform. According to the strategic choices of $P$ and $M$, their revenue changes are analyzed, and the model parameters are described.

(1) When $P$ adopts strategy $S_{P}^{1}$, it will share the access cost and scale revenue with $M$. At this time, regardless of the strategy $M$ adopts, $P$ can obtain the revenue of network externalities determined by the platform service price and the number of corporate customers. If $M$ adopts strategy $S_{M}^{1}$, it will receive transfer payments from $P$ and the price spillover revenue from the network externalities. When $P$ adopts strategy $S_{P}^{2}$, it will not share the access cost with $M$ but will monopolize the scale revenue. At this time, due to the decline of trust between the two parties, the basic customers of the platform will be lost, resulting in the decline of the revenue of network externalities. When $M$ adopts strategy $S_{M}^{1}$, regardless of which strategy is adopted, $P$ must pay a certain service cost.

(2) When $M$ adopts strategy $S_{M}^{1}$, it will obtain the valueadded business revenue and price spillover revenue of network externalities. At this time, if $P$ adopts strategy $S_{P}^{1}$, the access cost of $M$ will be shared with $P$, and $M$ will receive the shared scale revenue; otherwise, $M$ will bear the access cost alone, and the scale revenue will be monopolized by $P$. When $M$ adopts strategy $S_{M}^{2}$, if $P$ adopts strategy $S_{P}^{2}$, both will gain basic revenue, whereas if $P$ adopts strategy $S_{P}^{1}, M$ will face the risk of loss due to the improvement in the level of digitalization of other enterprises in the industry.

(3) Let $R_{P}$ and $T_{P}$ be the basic revenue and service revenue of the platform, respectively, and let $R_{M}$ and $T_{M}$ be the basic revenue and value-added revenue of the enterprise, respectively. Moreover, $\alpha$ is the distribution coefficient of the scale revenue, $\beta$ is the coefficient of sharing the access cost, $U$ is the scale revenue, $V$ is the access cost of the platform, $C_{S}$ is the service cost of the platform, $C_{M}$ is the service cost paid by $M$, and $\xi$ and $\eta$ are network externalities, the former of which is the quantity effect, and the latter of which is the price effect. Furthermore, let $N$ be the number of basic customers, let $Q$ be the loss of basic customers, and let $W$ be the risk of loss of $M$. The payoff matrix of $P$ and $M$ is presented in Table 1.

3.2. Construction of an Evolutionary Game Model. Let the probability of $P$ adopting strategy $S_{P}^{1}$ be $x(0 \leq x \leq 1)$, let the probability of $M$ adopting strategy $S_{M}^{1}$ be $y(0 \leq y \leq 1)$, and the corresponding expected revenues are $U_{P}^{1}$ and $U_{M}^{1}$, respectively. Then, the probability of $P$ adopting strategy $S_{P}^{2}$ is $1-x$, the probability of $M$ adopting strategy $S_{M}^{2}$ is $1-y$, and the corresponding expected revenues are $U_{P}^{2}$ and $U_{M}^{2}$, respectively. Assume that the expected revenues of the mixed strategy are $\bar{U}^{P}$ and $\bar{U}^{M}$, respectively. The following can then be determined according to Table 1.

The expected revenue of $P$ adopting strategy $S_{P}^{1}$ is as follows:

$$
\begin{aligned}
U_{P}^{1}= & y\left(R_{P}+T_{P}+\alpha U-(1-\beta) V-C_{S}+(\xi-\eta) N\right) \\
& +(1-y)\left(R_{P}+(\xi-\eta) N\right) \\
= & R_{P}+(\xi-\eta) N+y\left(T_{P}+\alpha U-C_{S}-(1-\beta) V\right) .
\end{aligned}
$$
follows:

The expected revenue of $P$ adopting strategy $S_{P}^{2}$ is as

$$
\begin{aligned}
U_{P}^{2}= & y\left(R_{P}+T_{P}+U-C_{S}+(\xi-\eta)(N-Q)\right) \\
& +(1-y)\left(R_{P}+(\xi-\eta)(N-Q)\right) \\
= & R_{P}+(\xi-\eta)(N-Q)+y\left(T_{P}+U-C_{S}\right) .
\end{aligned}
$$

The expected revenue of the two strategies of $P$ can be calculated as follows:

$$
\begin{aligned}
\bar{U}^{P}= & \left.((\alpha-1) U+(\beta-1) V) x+T_{P}+U-C_{S}\right) y \\
& +(\xi-\eta)(Q(x-1)+N)+R_{P} .
\end{aligned}
$$

According to the Malthusian dynamic equation [40], the growth rate of $P$ adopting strategy $S_{P}^{1}$ can be expressed by the difference between $U_{P}^{1}$ and $\bar{U}^{P}$. Let $t$ be the evolutionary time, and the replication dynamic equation of $P$ is then as follows:

$$
\begin{aligned}
F^{P}(x)= & \frac{\mathrm{d} x}{\mathrm{~d} t}=x\left(U_{P}^{1}-\bar{U}^{P}\right)=x(1-x)(((\alpha-1) U \\
& +(\beta-1) V) y+(\xi-\eta) Q) .
\end{aligned}
$$

Similarly, the expected revenue of $M$ adopting strategy $S_{M}^{1}$ is as follows:

$$
\begin{aligned}
U_{M}^{1}= & x\left(R_{M}+T_{M}+(1-\alpha) U-\beta V-C_{M}+\eta N\right) \\
& +(1-x)\left(R_{M}+T_{M}-V-C_{M}+\eta(N-Q)\right) \\
= & T_{M}+R_{M}-C_{M}-V+\eta(N-Q)+x(\eta Q \\
& +(1-\beta) V+(1-\alpha) U) .
\end{aligned}
$$
follows:

The expected revenue of $M$ adopting strategy $S_{M}^{2}$ is as

$$
U_{M}^{2}=x\left(R_{M}-W\right)+(1-x) R_{M}=R_{M}-x W .
$$

The expected revenue of the two strategies of $M$ can then be calculated as follows:

$$
\begin{aligned}
\bar{U}^{M}= & ((\eta Q+(1-\beta) V+(1-\alpha) U+W) x+\eta(N-Q) \\
& \left.+T_{M}-V-C_{M}\right) y-x W+R_{M} .
\end{aligned}
$$

The replication dynamic equation of $M$ is then as follows:

$$
\begin{aligned}
F^{M}(y)= & y(1-y)((\eta Q+(1-\beta) V+(1-\alpha) U+W) x \\
& \left.+\eta(N-Q)+T_{M}-V-C_{M}\right) .
\end{aligned}
$$


TABLE 1: The payoff matrix.

\begin{tabular}{cccc}
\hline \multirow{2}{*}{ Parties } & & & $M$ \\
& & $S_{M}^{1}$ & $S_{M}^{2}$ \\
\hline \multirow{4}{*}{$P$} & $S_{P}^{1}$ & $R_{P}+T_{P}+\alpha U-(1-\beta) V-C_{S}+(\xi-\eta) N$ & $R_{P}+(\xi-\eta) N$ \\
& $S_{P}^{2}$ & $R_{M}+T_{M}+(1-\alpha) U-\beta V-C_{M}+\eta N$ & $R_{M}-W$ \\
& $R_{P}+T_{P}+U-C_{S}+(\xi-\eta)(N-Q)$ & $R_{P}+(\xi-\eta)(N-Q)$ \\
& $R_{M}+T_{M}-V-C_{M}+\eta(N-Q)$ & $R_{M}$ \\
\hline
\end{tabular}

Let $(1-\alpha) U+(1-\beta) V=\delta_{1}$ and $T_{M}-V-C_{M}=\delta_{2}$. The evolution game model of $P$ and $M$ can then be obtained as follows:

$$
\left\{\begin{array}{l}
F^{P}(x)=\frac{\mathrm{d} x}{\mathrm{~d} t}=x(1-x)\left(-\delta_{1} y+(\xi-\eta) Q\right) . \\
F^{M}(y)=\frac{\mathrm{d} y}{\mathrm{~d} t}=y(1-y)\left(\left(\eta Q+\delta_{1}+W\right) x+\eta(N-Q)+\delta_{2}\right) .
\end{array}\right.
$$

In equation (9), $F^{P}(x)$ and $F^{M}(y)$ represent the evolution rates of the industrial Internet platform adopting the value cocreation strategy and the manufacturing SMEs adopting the access strategy, respectively. According to equation (9), the improvement of network externality revenue will prompt the industrial Internet platform to adopt the strategy of value cocreation, while changes in the scale revenue and access costs will have the opposite effect. This is because adopting the value cocreation strategy will reduce the direct revenue of the platform, but it will also attract more enterprises to access the platform or will at least not lead to the loss of basic customers. Therefore, the speed of the evolution of the trend toward the adoption of a value cocreation strategy by the platform depends on the magnitude of the impacts of the two factors mentioned previously on the platform revenue. For manufacturing SMEs, the shared revenues of scale, value-added services, and network externality that can be obtained will increase the evolution speed of adopting access strategies, while the investment in digital transformation and the service costs of the platform will inhibit their motivation to access the platform. In addition, the risk of loss will also encourage SMEs to adopt the access strategy. Equation (9) reflects the evolutionary game mechanism of the strategies of an industrial Internet platform and manufacturing SMEs. Based on this, the evolutionary path and stability of the strategies of both parties can be analyzed.

\section{Evolution Path and Stability Analysis}

According to the stability theorem of differential equations, the stable point of the replicated dynamic equation should be located at the zero point, where the first-order derivative is less than zero; that is, it should satisfy the following equation:

$$
\left\{\frac{\mathrm{d} F(x)}{\mathrm{d} x}<0 \mid F(x)=0, x \in[0,1]\right\} .
$$

According to equation (10), the ESSs for the industrial Internet platform and manufacturing SMEs can be discussed.
4.1. Industrial Internet Platform. Considering the ESS of the industrial Internet platform, equation (11) can be calculated according to equation (9):

$$
\frac{\mathrm{d} F^{P}(x)}{\mathrm{d} x}=(1-2 x)\left(-\delta_{1} y+(\xi-\eta) Q\right) .
$$

Let $\bar{y}=(\xi-\eta) \mathrm{Q} / \delta_{1}$. When $y=\bar{y}, F^{P}(x) \equiv 0$, i.e., $x$ is in the equilibrium state. When $y \neq \bar{y}$, let $F^{P}(x)=0$; thus, it can be concluded that $x=0$ and $x=1$ are two equilibrium points. At this time, if $\delta_{1}<(\xi-\eta) Q$, then $\bar{y}>1$, i.e., $y<\bar{y}$. Because when $x=1, \mathrm{~d} F^{P}(x) / \mathrm{d} x<0$, according to equation $(10), x^{*}=1$ is an evolutionary stability point. In this case, the evolution and stability strategy of the industrial Internet platform is not affected by the strategic choice of the SMEs but depends on the transfer payment to the enterprise and the possible loss of the network externality revenue of the industrial Internet platform. The adoption of strategy $S_{P}^{2}$ by the platform will result in the loss of basic customers. When the loss of network externality revenue caused by strategy $S_{P}^{2}$ is greater than its transfer payment to enterprises, the ESS of the platform is $S_{p}^{1}$, which is not related to the strategies of the enterprises.

If $\delta_{1}>(\xi-\eta) Q$, then $0<\bar{y}<1$, and there are two situations in the strategic evolution of the platform: when $y>\bar{y}$, when $x=1, \mathrm{~d} F^{P}(x) / \mathrm{d} x<0$, then $x^{*}=0$ is the evolutionary stability point; when $y<\bar{y}$, when $x=1, \mathrm{~d} F^{P}(x) / \mathrm{d} x<0$, then $x^{*}=1$ is the evolutionary stability point. In this case, when the transfer payment of the platform is greater than its loss of network externality revenue, the probability of the SME choosing different strategies becomes the decisive factor of the ESS of the platform. When the probability of the enterprise adopting strategy $S_{M}^{1}$ is greater than the critical value $\bar{y}$, the expected revenue of the platform from adopting strategy $S_{P}^{2}$ will be greater than that from adopting strategy $S_{P}^{1}$, so $S_{P}^{2}$ will become the ESS, and, conversely, the ESS will be $S_{P}^{1}$.

In general, in the initial stage of development, the industrial Internet platform has a small number of customers, and the scale effect is not significant. The platform needs to improve services such as price subsidies, revenue-sharing, and cost-sharing to attract enterprises to access it. In this case, the values of $U$ and $V$ are larger, and it is easier to meet the condition of $\delta_{1}>(\xi-\eta) Q$. The lower the willingness of SMEs to access the platform, the greater the probability that the platform adopts the strategy of value cocreation. When the willingness of SMEs to access the platform increases to $(\xi-\eta) \mathrm{Q} / \delta_{1}$, the platform will change its strategy. With the increasing number of enterprises connected to the platform, the transfer payment of the platform will decrease; that is, the values of $U$ and $V$ will decrease. In addition, with the 
improvement of the level of digitalization of SMEs, the transfer costs between different platforms will be reduced, and the SMEs will be more sensitive to the service of the platform; this will also increase the risk of the churning of platform customers; that is, the value of $Q$ will increase. Therefore, the constraints will be changed to $\delta_{1}<(\xi-\eta) Q$, and the platform will certainly adopt the strategy of value cocreation.

4.2. Manufacturing SMEs. Considering the ESS of manufacturing SMEs, equation (12) can be calculated according to equation (8).

$$
\frac{\mathrm{d} F^{M}(y)}{\mathrm{d} y}=(1-2 y)\left(\left(\eta Q+\delta_{1}+W\right) x+\eta(N-Q)+\delta_{2}\right) \text {. }
$$

Let $\bar{x}=\left(-\delta_{2}-\eta(N-Q)\right) /\left(\eta Q+\delta_{1}+W\right)$. When $x=\bar{x}$, $F^{M}(y) \equiv 0$, i.e., $y$ is in the equilibrium state. When $x \neq \bar{x}$, let $F^{M}(y)=0$; thus, it can be concluded that $y=0$ and $y=1$ are two equilibrium points. At this time, if $\delta_{2}+\eta(N-Q)>0$, then $\bar{x}<x$. Because when $x=1$, $\mathrm{d} F^{P}(x) / \mathrm{d} x<0$, according to equation (10), $x^{*}=1$ is an evolutionary stability point. In this case, choosing strategy $S_{M}^{1}$ can always increase the revenue of enterprises, so their ESS will be $S_{M}^{1}$, which is unrelated to the platform strategy.

If $\delta_{2}+\eta(N-Q)<0$, when the platform adopts strategy $S_{P}^{1}$, the revenue of the enterprise from adopting strategy $S_{M}^{1}$ is greater than that from adopting strategy $S_{M}^{2}$; then, $\delta_{1}+\delta_{2}+\eta N+W>0$, i.e., $0<\bar{x}<1$. There are two situations in the strategic evolution of SMEs: when $x>\bar{x}$, when $y=1$, $\mathrm{d} F^{M}(y) / \mathrm{d} y<0$, then $y^{*}=1$ is the evolutionary stability point; when $x<\bar{x}$, when $y=1, \mathrm{~d} F^{M}(y) / \mathrm{d} y<0$, then $y^{*}=0$ is the evolutionary stability point. In these two cases, the probability that the platform adopts strategy $S_{P}^{1}$ is the key to determining the ESS of the enterprises. If the probability that the platform will adopt strategy $S_{P}^{1}$ is greater than the critical value $\bar{x}$, the expected revenue of enterprises from adopting strategy $S_{M}^{1}$ will be greater than that from adopting strategy $S_{M}^{2}$, so $S_{M}^{1}$ will become an evolutionarily stable strategy; otherwise, the evolutionarily stable strategy will be $S_{M}^{2}$.

Generally speaking, the cost of enterprises to access the platform is huge in the initial stage, i.e., $\delta_{2}+\eta(N-Q)<0$. The strategic choice of enterprises depends heavily on whether the platform adopts the strategy of value cocreation to share their high access cost. With the improvement of the level of digitalization of enterprises, their operating revenue increases, and their investment decreases, which satisfies the constraint of $\delta_{2}+\eta(N-Q)>0$. At this time, regardless of which strategy the platform adopts, the enterprises will adopt the access strategy.

\section{Analysis of the Interaction between the Two Parties}

In the previous section, the evolution paths and stability strategies of the industrial Internet platform and manufacturing SMEs under different constraints were analyzed. Subsequently, the ESS and the equilibrium state of the system under the interaction of both parties are discussed. According to Friedman's findings [41], the ESS can be obtained by analyzing the local stability of the Jacobian matrix of the system.

Let $F^{P}(x)=0$ and $F^{M}(y)=0$, and it can be concluded that there are five local equilibrium points: $E_{1}(0,0), E_{2}(0,1)$, $E_{3}(1,0), E_{4}(1,1)$, and $E_{5}\left(x^{*}, y^{*}\right)$, where $x^{*}=\left(-\delta_{2}-(N-\right.$ Q) $\eta) /\left(\eta Q+W+\delta_{1}\right)$ and $y^{*}=(\xi-\eta) Q / \delta_{1}$. The stability of the equilibrium points can be judged by the rank and trace of the Jacobian matrix. According to equation (9), the Jacobian matrix of the system is as follows.

$$
J=\left(\begin{array}{cc}
\frac{\partial F^{R}(x)}{\partial x} & \frac{\partial F^{R}(x)}{\partial y} \\
\frac{\partial F^{M}(y)}{\partial x} & \frac{\partial F^{M}(y)}{\partial y}
\end{array}\right)
$$

In equation (13), $\partial F^{R}(x) / \partial x=(1-2 x)\left(-\delta_{1} y+\right.$ $(\xi-\eta) Q), \partial F^{M}(y) / \partial y=(1-2 y)\left(\left(\eta Q+\delta_{1}+W\right) x+\eta(N-\right.$ $\left.Q)+\delta_{2}\right), \partial F^{R}(x) / \partial y=-\delta_{1} x(1-x)$, and $\partial F^{M}(y) / \partial x=$ $y(1-y)\left(\delta_{1}+\eta Q+W\right)$. The rank and trace are, respectively, defined as follows:

$$
\begin{aligned}
\operatorname{det}(J)= & (1-2 x)\left(-\delta_{1} y+(\xi-\eta) Q\right)(1-2 y) \\
& \left(\left(\eta Q+\delta_{1}+W\right) x+(N-Q) \eta+\delta_{2}\right) \\
& +\delta_{1} x(1-x) y(1-y)\left(\delta_{1}+\eta Q+W\right), \\
\operatorname{tr}(J)= & (1-2 x)\left(-\delta_{1} y+(\xi-\eta) Q\right)+(1-2 y) \\
& \left(\left(\eta Q+\delta_{1}+W\right) x+(N-Q) \eta+\delta_{2}\right) .
\end{aligned}
$$

The definitions of $\operatorname{det}(J)$ and $\operatorname{tr}(J)$ corresponding to the five equilibrium points are reported in Table 2 .

The ESS requires $\operatorname{det}(J)>0$ and $\operatorname{tr}(J)<0$ at the corresponding equilibrium points. According to the local stability analysis of each equilibrium point, the stability results under four different conditions were obtained and are reported in Table 3.

According to the stability analysis results of the equilibrium points, the strategic evolution process of the platform and enterprises can be determined. The stability results of the system evolution strategy under the four different conditions are subsequently analyzed and discussed.

(1) According to Table 3, the evolutionary stability points of cases 1 and 3 are both $(1,1)$, the corresponding ESSs are $\left(S_{P}^{1}, S_{M}^{1}\right)$, and the unstable points are $(0,0)$ and $(0,1)$. In case $1, S_{P}^{1}$ and $S_{M}^{1}$ are the dominant strategies corresponding to $P$ and $M$, respectively. The system cannot reach evolutionary stability at point $(0,0)$ and will evolve to point $(1$, $1)$. In case 3 , when the platform adopts strategy $S_{P}^{2}$, the revenue of enterprises with strategy $S_{M}^{2}$ is greater than that of enterprises with strategy $S_{M}^{1}$, so the system will not reach evolutionary stability at point $(0,1)$ but will evolve toward point $(0,0)$. Because $(\xi-\eta) Q>\delta_{1}, S_{P}^{1}$ is still the ultimate strategy of the platform. Therefore, according to 
TABLE 2: The definitions of $\operatorname{det}(J)$ and $\operatorname{tr}(J)$ of each equilibrium point.

\begin{tabular}{lcc}
\hline $\begin{array}{l}\text { Equilibrium } \\
\text { point }\end{array}$ & $\operatorname{det}(J)$ & $\operatorname{tr}(J)$ \\
\hline$E_{1}(0,0)$ & $(\xi-\eta) Q\left(\delta_{2}+\eta(N-Q)\right)$ & $(\xi-\eta) Q+\delta_{2}+\eta(N-Q)$ \\
$E_{2}(0,1)$ & $\left(\delta_{1}-(\xi-\eta) Q\right)\left(\delta_{2}+\eta(N-Q)\right)$ & $\left((\xi-\eta) Q-\delta_{1}\right)-\left(\delta_{2}+\eta(N-Q)\right)$ \\
$E_{3}(1,0)$ & $-(\xi-\eta) Q\left(\delta_{1}+W+\eta N+\delta_{2}\right)$ & $-(\xi-\eta) Q+\delta_{1}+W+\eta N+\delta_{2}$ \\
$E_{4}(1,1)$ & $\left(-\delta_{1}+(\xi-\eta) Q\right)\left(\delta_{1}+W+\eta N+\delta_{2}\right)$ & $\left(\delta_{1}-(\xi-\eta) Q\right)-\left(\delta_{1}+W+\eta N+\delta_{2}\right)$ \\
$E_{5}\left(x^{*}, y^{*}\right)$ & $-\left(\delta_{2}+\eta(N-Q)\right)(\xi-\eta) Q\left(1+\left(\delta_{2}+\eta(N-Q) / \eta Q+W+\delta_{1}\right)\right)\left(1-\left((\xi-\eta) Q / \delta_{1}\right)\right)$ & 0 \\
\hline
\end{tabular}

TABLE 3: The local stability of each equilibrium point under four conditions.

\begin{tabular}{|c|c|c|c|c|}
\hline Case & Equilibrium point & $\operatorname{det}(J)$ & $\operatorname{tr}(J)$ & Stability state \\
\hline (1) $(\xi-\eta) Q>\delta_{1}, \eta(Q-N)<\delta_{2}$ & $\begin{array}{l}E_{1}(0,0) \\
E_{2}(0,1) \\
E_{3}(1,0) \\
E_{4}(1,1)\end{array}$ & $\begin{array}{l}+ \\
- \\
- \\
+\end{array}$ & $\begin{array}{l}+ \\
-\end{array}$ & $\begin{array}{c}\text { Unstable point } \\
\text { Saddle point } \\
\text { Saddle point } \\
\text { ESS }\end{array}$ \\
\hline (2) $(\xi-\eta) Q<\delta_{1}, \eta(Q-N)<\delta_{2}$ & $\begin{array}{l}E_{1}(0,0) \\
E_{2}(0,1) \\
E_{3}(1,0) \\
E_{4}(1,1)\end{array}$ & $\begin{array}{l}+ \\
+ \\
- \\
-\end{array}$ & $\begin{array}{l}+ \\
-\end{array}$ & $\begin{array}{c}\text { Unstable point } \\
\text { ESS } \\
\text { Saddle point } \\
\text { Saddle point }\end{array}$ \\
\hline (3) $(\xi-\eta) Q>\delta_{1}, \eta(Q-N)>\delta_{2}$ & $\begin{array}{l}E_{1}(0,0) \\
E_{2}(0,1) \\
E_{3}(1,0) \\
E_{4}(1,1)\end{array}$ & $\begin{array}{l}- \\
+ \\
- \\
+\end{array}$ & $\begin{array}{l}+ \\
-\end{array}$ & $\begin{array}{c}\text { Saddle point } \\
\text { Unstable point } \\
\text { Saddle point } \\
\text { ESS }\end{array}$ \\
\hline (4) $(\xi-\eta) Q<\delta_{1}, \eta(Q-N)>\delta_{2}$ & $\begin{array}{c}E_{1}(0,0) \\
E_{2}(0,1) \\
E_{3}(1,0) \\
E_{4}(1,1) \\
E_{5}\left(x^{*}, y^{*}\right)\end{array}$ & $\begin{array}{l}- \\
- \\
- \\
- \\
+\end{array}$ & 0 & $\begin{array}{l}\text { Saddle point } \\
\text { Saddle point } \\
\text { Saddle point } \\
\text { Saddle point } \\
\text { Central point }\end{array}$ \\
\hline
\end{tabular}

Note. $0<x^{*}<1$ and $0<y^{*}<1$ are satisfied only in Case 4 .

Section 2.2, as the probability of the platform choosing strategy $S_{P}^{1}$ gradually exceeds the value of $\left(-\delta_{2}-\eta(N-Q)\right) /\left(\eta Q+\delta_{1}+W\right)$, the enterprises will eventually choose strategy $S_{M}^{1}$; that is, the system will achieve evolutionary stability at point $(1,1)$. In both cases, the choice of the platform's strategy plays a decisive role in the evolution of the system.

(2) In case 2 , the evolutionary stability point is $(0,1)$, the corresponding ESS is $\left(S_{P}^{2}, S_{M}^{1}\right)$, and the unstable point is $(0,0)$. In this case, when $\delta_{1}>(\xi-\eta) Q$, the strategic evolution of the platform will be affected by the enterprises. When $\eta(Q-N)<\delta_{2}$, the dominant strategy of the enterprises is $S_{M}^{1}$, so the system will not reach evolutionary stability at point $(0,0)$ but will evolve to point $(0,1)$. Furthermore, according to Section 2.1, when the probability of the enterprises choosing strategy $S_{M}^{1}$ is greater than the value of $(\xi-\eta) Q / \delta_{1}$, the ESS of the platform is $S_{P}^{2}$; that is, the system will reach evolutionary stability at point $(0,1)$. In this case, the strategic choice of the enterprises plays a decisive role in the evolution of the system.

(3) In case (4), there is no evolutionary stability point, and the system evolves as a result of rotation around a central fixed point $\left(x^{*}, y^{*}\right)$ along a closed orbit but is not focused. In this case, if the initial strategy of the platform is $S_{P}^{1}$, the strategy of the enterprises will evolve toward $S_{M}^{1}$. With the increase of the evolution time, the number of enterprises adopting strategy $S_{M}^{1}$ will increase. When its group ratio exceeds the critical value $(\xi-\eta) Q / \delta_{1}$, the strategy of the platform will evolve to $S_{P}^{2}$. Similarly, when the group ratio of the platform adopting strategy $S_{P}^{2}$ exceeds the critical value $\left(-\delta_{2}-\eta(N-Q)\right) /\left(\eta Q+\delta_{1}+W\right)$, the enterprise strategy will evolve to $S_{M}^{2}$. In this case, the strategic evolution of the system is a cyclic process that cannot reach stability.

Case 4 reflects the uncertainty of the game result between the industrial Internet platform and manufacturing SMEs. The initial state of the system determines the difference between the game result at a given moment and the position of the central fixed point, which in turn depends on the judgment of the parties about their own gains. Therefore, it is critical to improve the expected revenue based on the cooperation between the platform and enterprises.

Considering the current reality of industrial Internet development, subsidies for platforms that adopt the cocreation strategy or enterprises that adopt the access strategy are considered to improve the evolutionary outcome of the system. Assume that $F(F>0)$ is the subsidy for the platform, and $K(K>0)$ is the subsidy for the enterprises. An evolutionary game model under the subsidy incentive can then be established, as given by the following equation: 


$$
\left\{\begin{array}{l}
\frac{\mathrm{d} x}{\mathrm{~d} t}=x(1-x)\left(-\delta_{1} y+(\xi-\eta) Q+F\right), \\
\frac{\mathrm{d} y}{\mathrm{~d} t}=y(1-y)\left(\left(\eta Q+\delta_{1}+W\right) x+(N-Q) \eta+\delta_{2}+K\right) .
\end{array}\right.
$$

The purpose of improving the system is to promote cooperation between the platform and enterprises, i.e., to make the system achieve evolutionary stability at point $(1,1)$. Let the Jacobian matrix corresponding to equation (12) be $J^{*}$; then, the rank and trace of $J^{*}$ are $\operatorname{det}\left(J^{*}\right)=$ $\left(-\delta_{1}+(\xi-\eta) Q+F\right)\left(\delta_{1}+W+N \eta+\delta_{2}+K\right)$ and $\operatorname{tr}\left(J^{*}\right)$ $=\left(\delta_{1}-(\xi-\eta) Q-F\right)-\left(\delta_{1}+W+N \eta+\delta_{2}+K\right)$, respectively. Because $(\xi-\eta) Q<\delta_{1}, \quad \eta(Q-N)>\delta_{2}$, and $\delta_{1}+\delta_{2}+\eta N+W>0$, to make $\operatorname{det}\left(J^{*}\right)>0, \operatorname{tr}\left(J^{*}\right)<0$, the subsidy given to the platform must be greater than the difference between the changes in revenue of the two strategies; that is, $F>\delta_{1}-(\xi-\eta) Q$ should be satisfied. Therefore, the improvement of case 4 only requires the consideration of the subsidy for the platform, and the strategy of the platform plays a decisive role in the result of system evolution.

\section{Simulation Analysis}

It is difficult for mathematical models of evolutionary games to dynamically and intuitively describe the process by which evolutionary strategies achieve equilibrium. Multiagent modeling and simulation technology can be used to simulate the evolutionary process and emergent phenomena of the system by defining individual behavioral rules and interaction mechanisms and can present the change process of the system at the macro-level [42]. To more intuitively demonstrate the evolution path and steady state of the system under different constraints and influencing factors, simulations of the evolution process of the system strategy were performed using the NetLogo simulation platform.

An agent-based model (ABM) of the strategic evolution between industrial Internet platforms and manufacturing SMEs was established on the NetLogo 6.0.4 platform. The number of platforms was set as 500, of which the numbers of those adopting strategies $S_{P}^{1}$ and $S_{P}^{2}$ were both 250. The number of SMEs was set as 1000, of which 500 adopted strategy $S_{M}^{1}$ and 500 adopted strategy $S_{M}^{2}$. P-S1 (red) and P-S2 (green) were set to represent the proportions of platforms adopting strategies $S_{P}^{1}$ and $S_{P}^{2}$, respectively. Moreover, M-S1 (blue) and M-S2 (yellow) were set to represent the proportions of enterprises adopting strategies $S_{M}^{1}$ and $S_{M}^{2}$, respectively. The basic parameters were set in the background, and the variable parameters were adjusted by rectangular sliders. The model simulation interface is shown in Figure 1.

Figure 1 presents the simulation interface on the NetLogo platform. By changing the values of the corresponding parameters, the evolutionary strategy analysis was verified by a simulation. According to the basic assumptions of the model and the constraint relationships among the parameters in the four cases, the assignment of the model parameters was as follows. In case $1, R_{P}=10, T_{P}=1$, $\alpha=0.6, \beta=0.4, U=1, V=5, C_{S}=0.5, \xi=0.4, \eta=0.2$, $N=300, \quad Q=50, \quad R_{M}=1, \quad T_{M}=0.3, \quad C_{M}=0.1, \quad$ and $W=0.1$. With the other parameters remaining unchanged, $V=40$ in case $2, V=30$ and $Q=100$ in case 3 , and $Q=100$ and $V=50$ in case 4 . The simulation duration was set to 50 units, and the simulation results are presented in Figure 2.

Figure 2 exhibits the evolutionary game process of the strategic choices of the industrial Internet platforms and manufacturing SMEs in the four cases. In cases 1, 2, and 3, the strategic evolution trends of the platforms and SMEs were relatively stable, and the strategic adjustment rate of the platforms was higher than that of the SMEs. In case 4, the numbers of individuals adopting the value cocreation strategy and the access strategy increased at the initial stage. When the proportion of individuals adopting the access strategy was greater than $0.658\left((\xi-\eta) Q / \delta_{1}\right)$, the number of individuals adopting the value cocreation strategy began to decline, and when the proportion decreased to 0.806 $\left(1-\left(\left(-\delta_{2}-\eta(N-Q)\right) /\left(\eta Q+\delta_{1}+W\right)\right)\right)$, the number of individuals adopting the access strategy also began to decline. When the proportion of individuals adopting the access strategy was less than 0.658 , the number of individuals adopting the access strategy increased. The strategies of the platforms and enterprises interacted with each other cyclically with time, and there was no stable strategy in the system.

A simulation of the system evolution after introducing subsidy incentives to case 4 was then conducted. With the other parameters remaining unchanged, $F=30, K=3$, and the constraint of $F>\delta_{1}-(\xi-\eta) Q$ was satisfied. According to equation (12), the interaction rules of individuals were set, and the simulation results are exhibited in Figure 3.

Figure 3 presents the improved simulation results of case 4 , from which it is evident that, after the introduction of subsidy incentives, the platforms adopted the value cocreation strategy, and the enterprises adopted the access strategy. The simulation results verify the correctness of the four case analyses and the effectiveness of the improvement due to the introduction of subsidy incentives to case 4 . In consideration of cases 1 and 2, the impacts of changes in various parameter values on the system evolution results were analyzed from three aspects, namely, the platform network externality, the churning of basic customers, and the cost-sharing ratio. According to Section 3, under specific constraints, the initial proportion of subjects with different strategies only affects the rate at which the system reaches equilibrium, and there is no difference in the system evolution results. Without losing generality, the initial probabilities of different strategies were, respectively, $x=0.5$ and $y=0.5$ in the simulation process.

6.1. Network Externality. The network externality parameters directly affect the revenue of network externality, thereby affecting the path of the strategic evolution of the system. Considering the constraint of the value of $\xi$, the evolution of the system strategy was simulated by keeping 


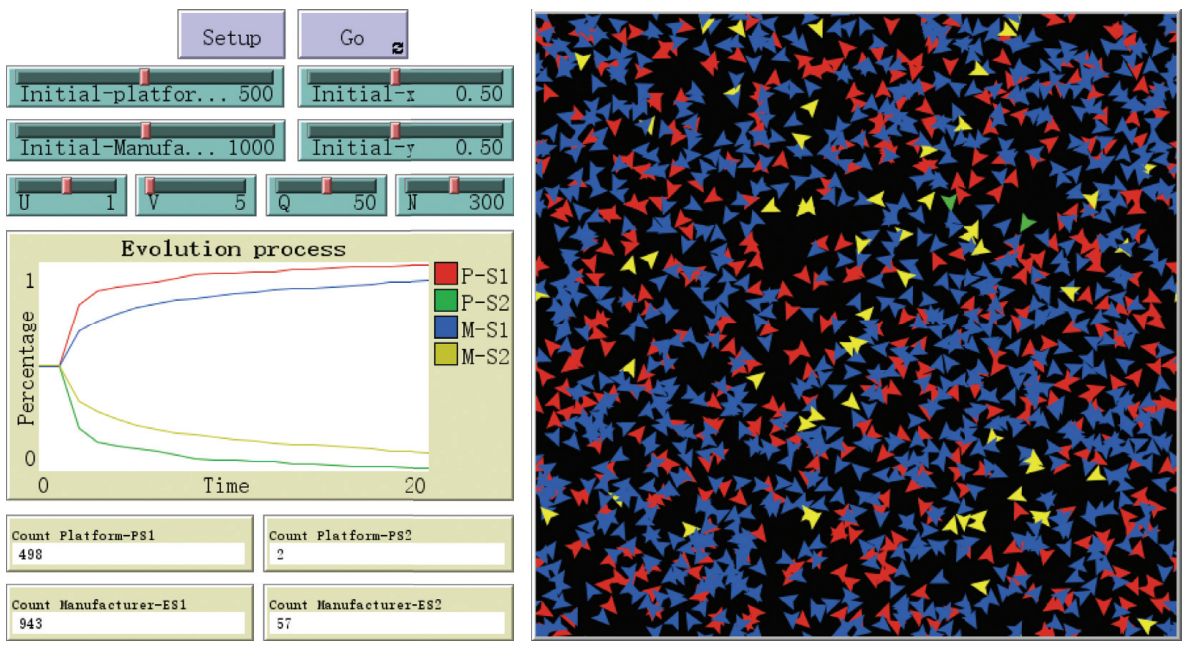

FIGURE 1: The simulation interface.

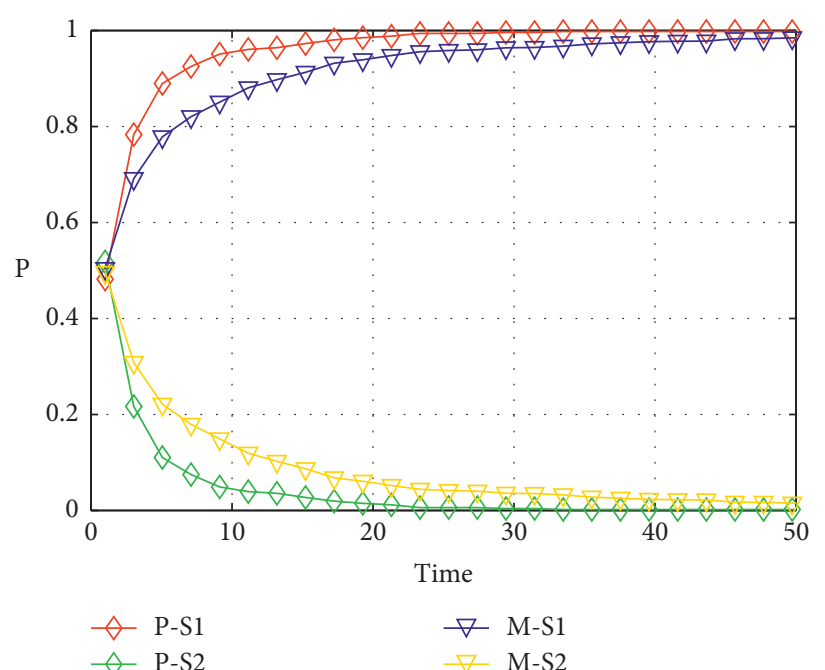

(a)

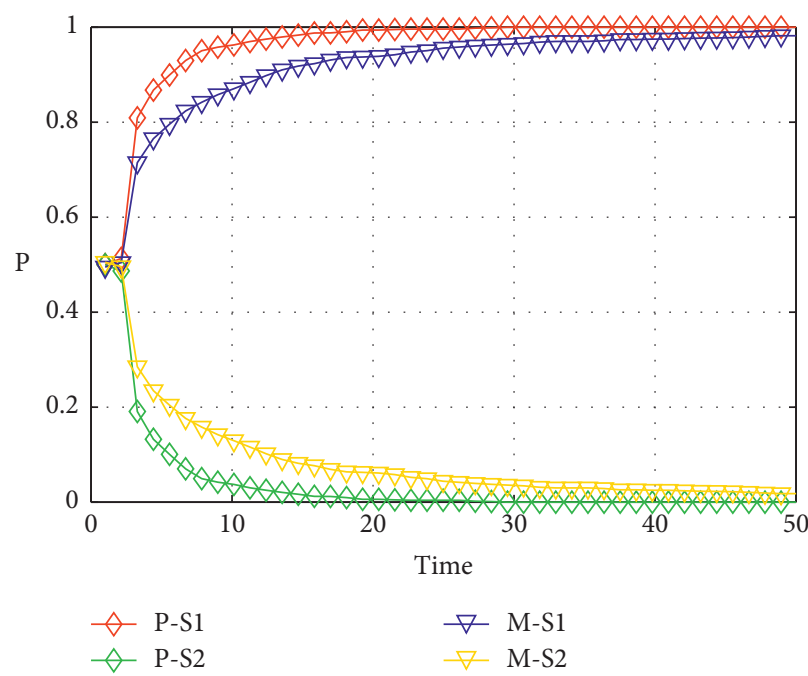

(c)

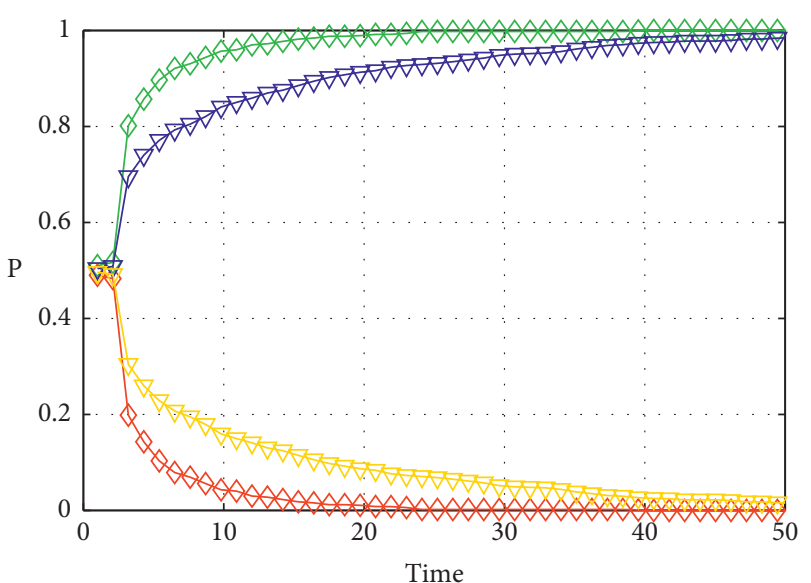

$$
\begin{aligned}
& \diamond \text { P-S1 } \\
& \diamond \text { P-S2 } \\
& \nabla \text { M-S1 } \\
& \nabla \text { M-S2 }
\end{aligned}
$$

(b)

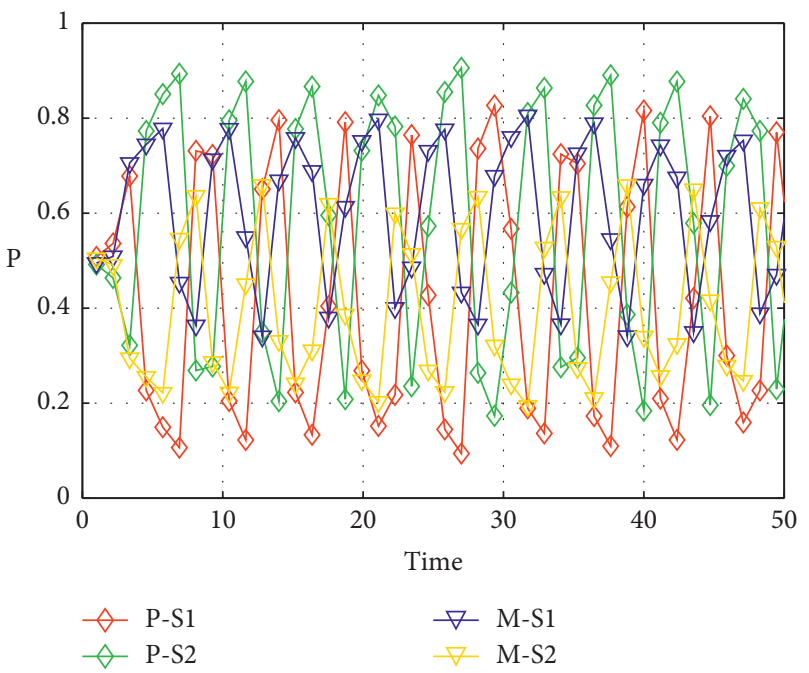

(d)

Figure 2: The simulation results. (a) Case 1. (b) Case 2. (c) Case 3. (d) Case 4. 


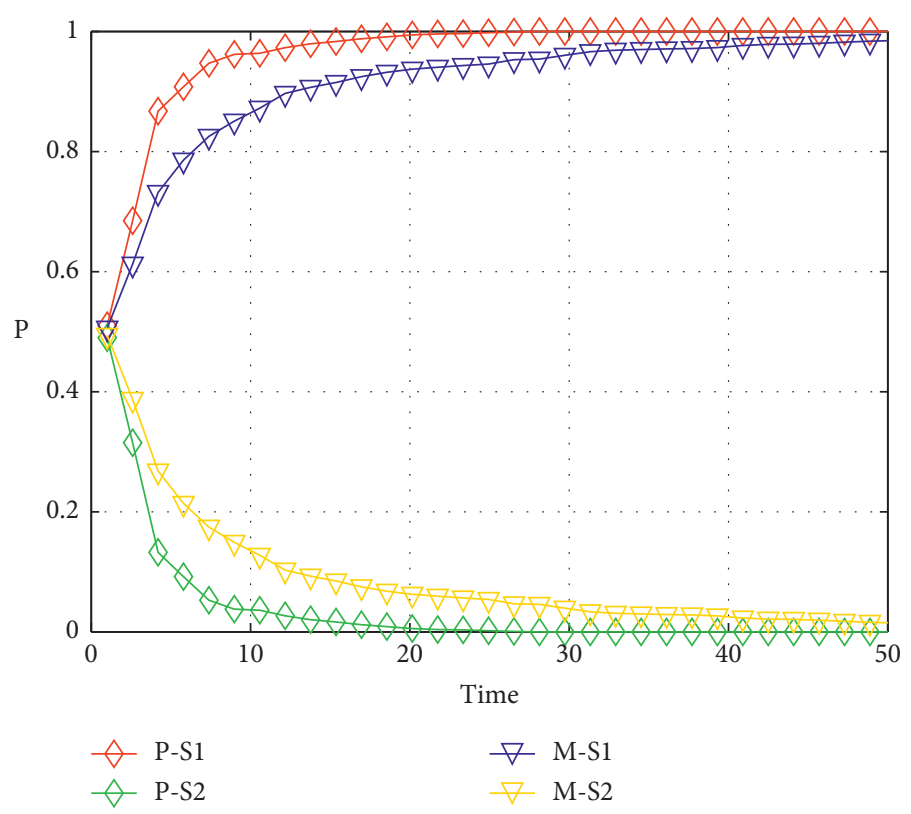

(a)

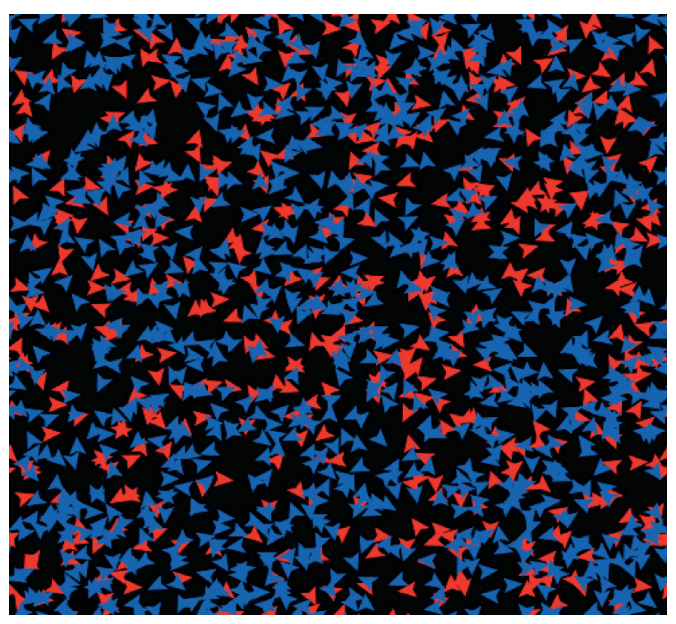

(b)

Figure 3: The improved simulation results of Case 4. (a) The process of strategy evolution. (b) Results of the group strategy evolution.

the other parameters constant and changing the value of $\xi$. The results are presented in Figure 4.

Figure 4 shows the influence of the network externalities of the platforms on the strategic evolution of the system. According to Figure 4(a), with the increase of $\xi$, the time required for the platforms to achieve evolutionary stability was greatly shortened, but the strategic adjustment rate was gradually reduced. This demonstrates that, in case 1 , the greater the network externality, the stronger the willingness of platforms to adopt the value cocreation strategy, but the incremental effect was gradually weakened. Affected by the changes in the platform strategy, the speed of enterprises moving toward the access strategy was found to be slightly improved.

According to Figure 4(b), with the increase of network externality, the time required for the platform to reach the ESS increased, but the value cocreation strategy was ultimately not adopted, and the change of $\xi$ had little impact on the enterprise strategy. This is because, in case 2, the SMEs always gained additional revenue by adopting the access strategy, and the quantitative effect of the network externality of the platforms did not affect the outcome of their strategic choice. For the platforms, their ultimate choice was to not adopt the value cocreation strategy. However, the strategy adjustment rate of the platforms decreased significantly due to the loss of basic customers, and the larger the value of $\xi$, the greater the customer loss.

6.2. Churning of Basic Customers. When platforms adopt the value cocreation strategy, the motivation of enterprises to access the platforms increases, and, conversely, the platforms will lose their basic customers. Considering the constraint of the value of $Q$, the evolution of the system strategy was simulated by keeping the other parameters constant and changing the value of $Q$. The results are shown in Figure 5.

Figure 5 demonstrates the influence of the churning of basic customers of the platforms on the strategic evolution of the system. According to Figure 5(a), with the gradual increase of $Q$, the evolution speed of the platforms adopting the value cocreation strategy tended to notably increase. This is because the loss of basic customers will directly affect the revenue of platforms. To prevent the increase of customer loss from bringing more losses, the platforms will actively adopt the value cocreation strategy. This also shows that if enterprises are sensitive to the distribution of cooperation revenue, or if the cost of transferring between different platforms is low, it will be beneficial to promote platforms to adopt the value cocreation strategy.

According to Figure 5(b), the increase of $Q$ was found to prolong the time required for the platform strategy to reach evolutionary stability and also had a slight negative impact on the adjustment speed of the strategy of enterprises. This is because the loss of basic customers will reduce the network externality revenue of both the platforms and enterprises. Therefore, with the increase of customer churn, the adjustment rate of the strategies of both parties will decrease relatively, thereby prolonging the time required for the system to achieve equilibrium.

6.3. Cost-Sharing Ratio. Cost-sharing can effectively reduce the access threshold of a platform, thereby increasing the motivation and feasibility of enterprises to access the platform. Considering the constraint of the value of $\beta$, the evolution of the system strategy was simulated by keeping the other parameters constant and changing the value of $\beta$. The results are presented in Figure 6. 


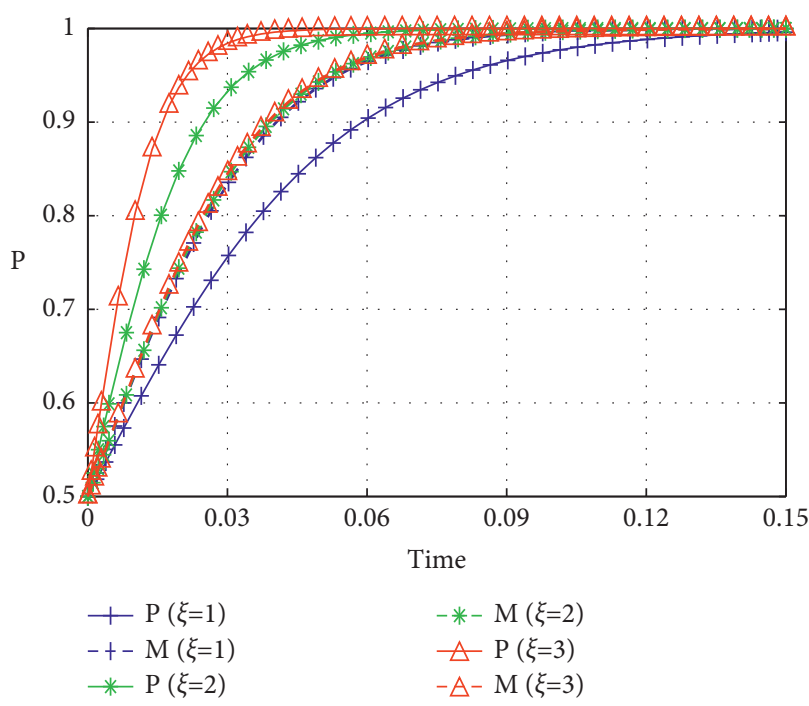

(a)

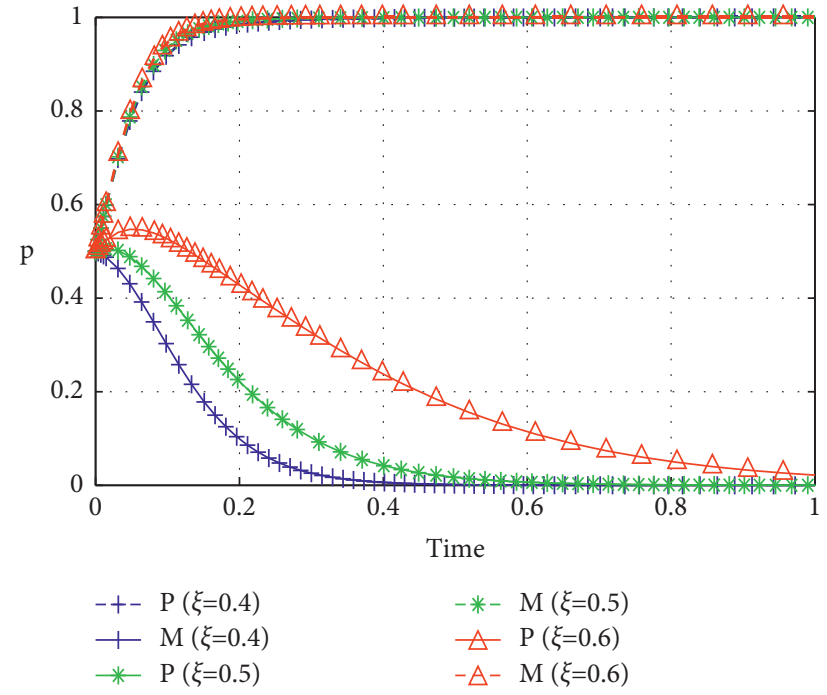

(b)

FIGURE 4: The impacts of network externality in different cases. (a) Case 1. (b) Case 2.

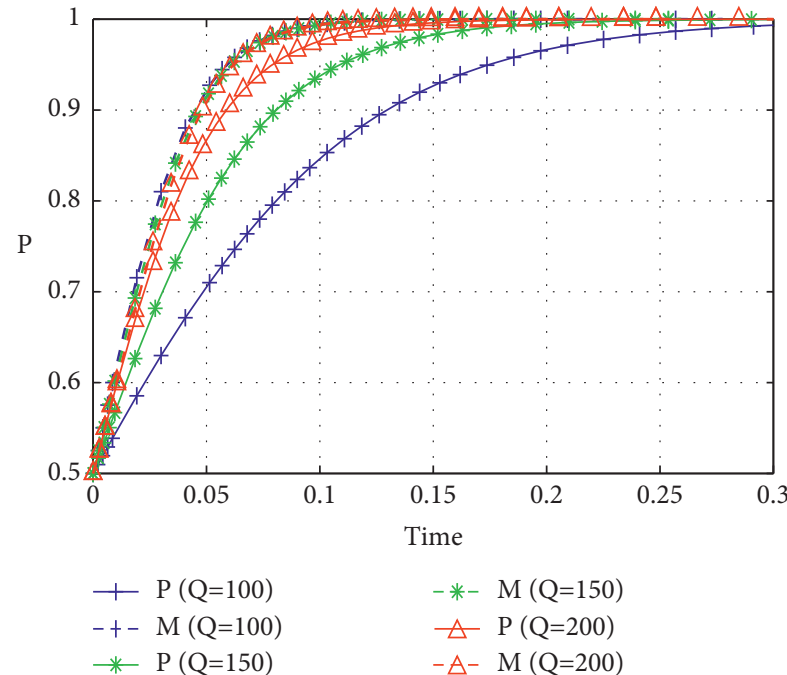

(a)

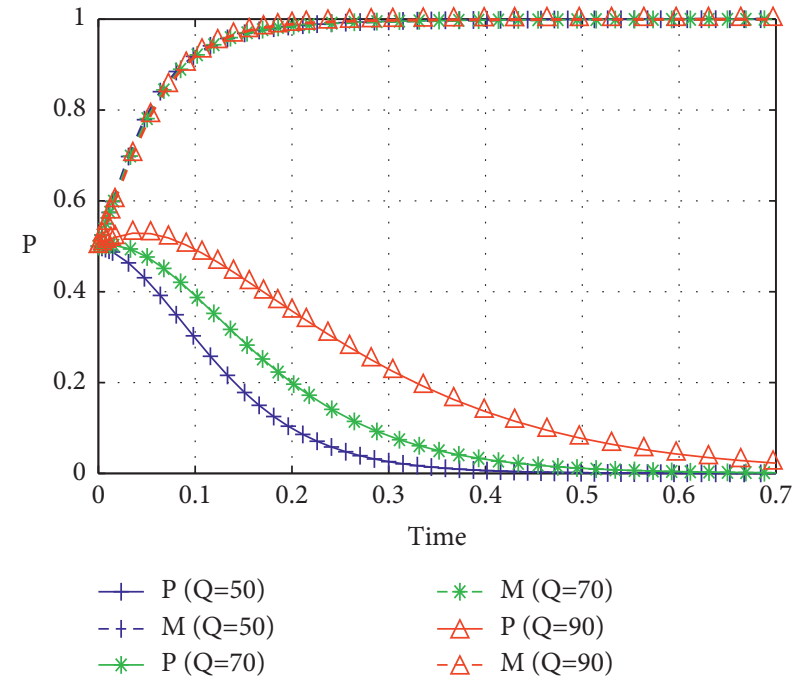

(b)

Figure 5: The impacts of the churning of basic customers in different cases. (a) Case 1. (b) Case 2.

Figure 6 reveals the influence of the cost-sharing ratio of platforms on the strategic evolution of the system. According to Figure 6(a), with the increase of the value of $\beta$, i.e., with the decrease of the cost-sharing ratio of the platform, the proportion of platforms adopting the value cocreation strategy increased significantly, while the strategies of the enterprises remained almost unchanged. This is because, in case 1, the increase in revenue from the enterprises' access to the platforms was greater than the cost borne by the enterprises, and the change in $\beta$ had a limited impact on the cost paid by the platforms when the value of $V$ was low. The strategy of the platforms mainly depends on the comparison between the network externality revenue and their transfer payments to enterprises. When the values of $(\xi-\eta) Q$ and $\delta_{1}$ are similar, the change in $\beta$ has a great influence.

According to Figure 6(b), the strategy adjustment rate of the platforms decreased with the increase of the value of $\beta$. This is because, in case 2 , the platforms did not adopt the value cocreation strategy, and the higher the cost-sharing ratio, the lower their willingness. For enterprises, with the increase in $\beta$, i.e., with the increase in cost, their willingness to access the platforms was found to decrease, so the time required to reach the evolutionary stability equilibrium was prolonged. 


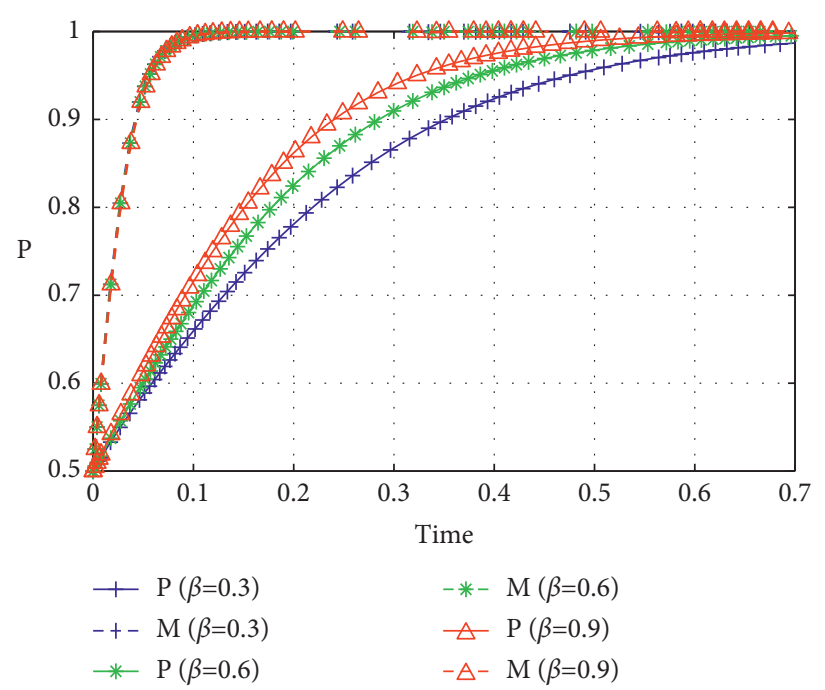

(a)

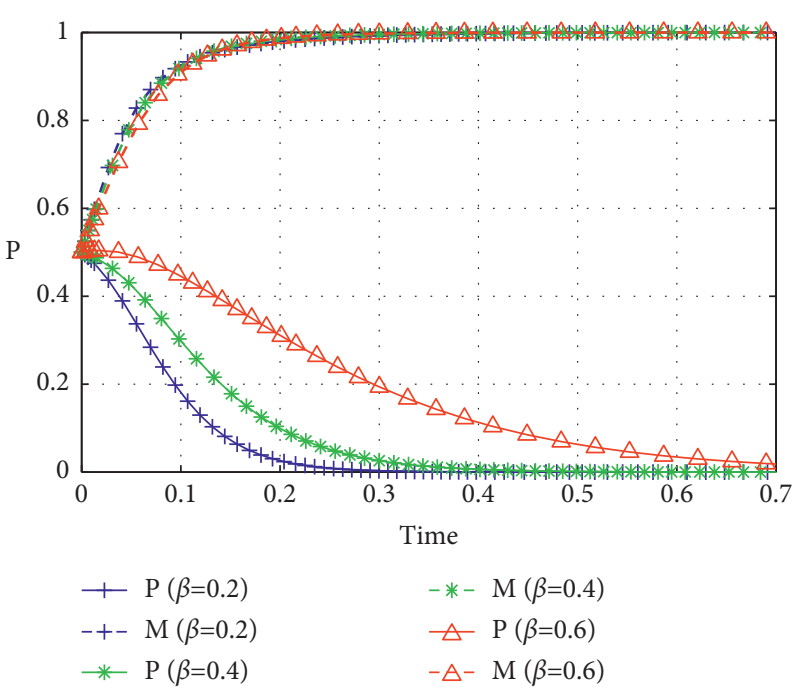

(b)

FIgURE 6: The impacts of the cost-sharing ratio in different cases. (a) Case 1. (b) Case 2.

\section{Conclusions}

To promote the empowerment of manufacturing SMEs by industrial Internet platforms, this work proposed a value cocreation cooperation mechanism based on the analysis of the real needs and dilemmas of both parties. An evolutionary game model was established, and the evolutionary stability strategies (ESSs) of individuals and the evolutionary equilibrium states of the system under different situations were determined. In combination with numerical examples, the impacts of the platform network externality, the churning of basic customers, and the cost-sharing ratio on the process of reaching the evolutionary equilibrium in the system were explored. The main conclusions and insights of this research are as follows:

(1) The key factors influencing the choice of platform strategy are different from those of manufacturing SMEs. The strategic choice of platforms is mainly influenced by transfer payments, network externality, and the willingness of enterprises to access the platform. The strategic choice of enterprises is mainly influenced by the change in revenue from accessing the platform, the risk of loss from not accessing the platform, and the willingness of the platform to adopt a value cocreation strategy. In the early stage of cooperation, the strategic choice of enterprises depends largely on the willingness of the platform to adopt a value cocreation strategy, which it will do in the later stage, as the market competition intensifies, enterprises become more digital, and the risk of losing customers increases. In addition, as the scale of customers increases, the platform network externality is enhanced, and enterprises will actively access the platform even if the platform no longer adopts a value cocreation strategy.
(2) There are four scenarios of system evolution depending on the strength of the platform transfer payments, the loss of network externality revenue, and the revenue that enterprises can obtain if the platform does not adopt a cocreation strategy. When the loss of network externality revenue is high, the dominant strategy of the platform is to adopt a value cocreation strategy. Under the influence of the platform strategy, enterprises will choose to access the platform, and the system will reach a desirable evolutionary steady state. When the loss of the network externality revenue of the platform is less than its transfer payments, if the enterprises can still obtain the revenue even if the platform does not adopt a value cocreation strategy, they will choose to access the platform, and the platform will not adopt a value cocreation strategy; conversely, the system will not achieve evolutionary stability, and the platform will need to be subsidized.

(3) It is crucial to enhance the expected cooperation between industrial Internet platforms and manufacturing SMEs. Due to the high cost and data security risks, SMEs have insufficient incentive or ability to access platforms. If a platform can adopt a value cocreation strategy, the motivation of enterprises to access the platform will be greatly increased, and the platform can rapidly expand its scale. However, transfer payments to enterprises affect the incentive for platforms to adopt a value cocreation strategy, and, therefore, platforms should be subsidized in the early stage of cooperation. For SMEs, the strength of transfer payments from the platform and risk of industry loss have greater impacts on their strategic choice in the early stage of cooperation, while network externalities will become their focus as 
their level of digitalization increases. As the scale of customers expands, and the level of digitalization of enterprises increases, both parties will cooperate spontaneously, and the empowering effect of the platform on enterprises will be promoted.

\section{Data Availability}

The simulation data used to support the findings of this study are included within the article.

\section{Conflicts of Interest}

The authors declare that they have no conflicts of interest.

\section{Acknowledgments}

This work was supported by National Social Science Foundation of China (Grant no. 18BJY009).

\section{References}

[1] H. S. Kang, J. Y. Lee, S. Choi et al., "Smart manufacturing: past research, present findings, and future directions," International Journal of Precision Engineering and ManufacturingGreen Technology, vol. 3, no. 1, pp. 111-128, 2016.

[2] W. Qin, S. Chen, and M. Peng, "Recent advances in industrial internet: insights and challenges," Digital Communications and Networks, vol. 6, no. 1, pp. 1-13, 2020.

[3] K. Menon, H. Kärkkäinen, T. Wuest, and J. P. Gupta, "Industrial internet platforms: a conceptual evaluation from a product lifecycle management perspective," Proceedings of the Institution of Mechanical Engineers - Part B: Journal of Engineering Manufacture, vol. 233, no. 5, pp. 1390-1401, 2019.

[4] M. Jovanovic, B. Lalic, A. Mas, and A.-L. Mesquida, "The Agile approach in industrial and software engineering project management," Istrazivanja i projektovanja za privredu, vol. 13, no. 4, pp. 213-216, 2015.

[5] H. Lasi, P. Fettke, H.-G. Kemper, T. Feld, and M. Hoffmann, "Industry 4.0," Business \& Information Systems Engineering, vol. 6, no. 4, pp. 239-242, 2014.

[6] L. Li, "China's manufacturing locus in 2025: with a comparison of "Made-in-China 2025" and "Industry 4.0"," Technological Forecasting and Social Change, vol. 135, pp. 66-74, 2018.

[7] A. Pareek, M. O. Pal, and M. N. Halkara, "IoT-a pathway to smart India-part 5 (consumer applications)," International Journal On Future Revolution in Computer Science \& Communication Engineering, vol. 5, no. 2, pp. 67-71, 2019.

[8] S. Shan, X. Wen, Y. Wei, Z. Wang, and Y. Chen, "Intelligent manufacturing in industry 4.0: a case study of Sany heavy industry," Systems Research and Behavioral Science, vol. 37, no. 4, pp. 679-690, 2020.

[9] J. Li, J.-J. Qiu, Y. Zhou, S. Wen, K.-Q. Dou, and Q. Li, “Study on the reference architecture and assessment framework of industrial internet platform," IEEE Access, vol. 8, pp. 164950-164971, 2020.

[10] F. Núñez, S. Langarica, P. Díaz, M. Torres, and J. C. Salas, "Neural network-based model predictive control of a paste thickener over an industrial internet platform," IEEE Transactions on Industrial Informatics, vol. 16, no. 4, pp. 2859-2867, 2019.
[11] K. Menon, H. Kärkkäinen, and T. Wuest, "Industrial internet platform provider and end-user perceptions of platform openness impacts," Industry \& Innovation, vol. 27, no. 4, pp. 363-389, 2020.

[12] J. M. Müller, "Business model innovation in small-and medium-sized enterprises: strategies for industry 4.0 providers and users," Journal of Manufacturing Technology Management, vol. 30, 2019.

[13] C. Wang, L. Song, and S. Li, "The industrial internet platform: trend and challenges," Chinese Journal of Engineering Science, vol. 20, no. 2, pp. 15-19, 2018.

[14] M. Ehret and J. Wirtz, "Unlocking value from machines: business models and the industrial internet of things," Journal of Marketing Management, vol. 33, no. 1-2, pp. 111-130, 2017.

[15] S. S. Teh and D. M. H. Kee, "The readiness of small and medium enterprises for the industrial revolution 4.0," GATR Global Journal of Business Social Sciences Review, vol. 7, no. 4, pp. 217-223, 2019.

[16] J. Stentoft, K. Adsbøll Wickstrøm, K. Philipsen, and A. Haug, "Drivers and barriers for Industry 4.0 readiness and practice: empirical evidence from small and medium-sized manufacturers," Production Planning \& Control, vol. 32, no. 10, pp. 811-828, 2020.

[17] A. Bonamigo and C. G. Frech, "Industry 4.0 in services: challenges and opportunities for value co-creation," Journal of Services Marketing, vol. 35, 2020.

[18] D. Helbing, D. Brockmann, T. Chadefaux et al., "Saving human lives: what complexity science and information systems can contribute," Journal of Statistical Physics, vol. 158, no. 3, pp. 735-781, 2014.

[19] X. Zhang and X. Ming, "A comprehensive industrial practice for industrial internet platform (IIP): general model, reference architecture, and industrial verification," Computers \& Industrial Engineering, vol. 158, Article ID 107426, 2021.

[20] J. Li, J. J. Qiu, and K. Q. Dou, "Research on the reference architecture, core function and application value of industrial internet platform," Manufacturing Automation, vol. 40, pp. 103-106, 2018.

[21] P. K. Malik, R. Sharma, R. Singh et al., "Industrial internet of things and its applications in industry 4.0: state of the art," Computer Communications, vol. 166, pp. 125-139, 2021.

[22] J.-Q. Li, F. R. Yu, G. Deng, C. Luo, Z. Ming, and Q. Yan, "Industrial internet: a survey on the enabling technologies, applications, and challenges," IEEE Communications Surveys \& Tutorials, vol. 19, no. 3, pp. 1504-1526, 2017.

[23] C. Arnold, D. Kiel, and K. Voigt, "How the industrial internet of things changes business models in different manufacturing industries," International Journal of Innovation Management, vol. 20, no. 8, Article ID 1640015, 2016.

[24] D. Kiel, C. Arnold, and K.-I. Voigt, "The influence of the Industrial Internet of Things on business models of established manufacturing companies-a business level perspective," Technovation, vol. 68, pp. 4-19, 2017.

[25] Y. Zhang, P. Zhang, F. Tao, Y. Liu, and Y. Zuo, "Consensus aware manufacturing service collaboration optimization under blockchain based Industrial Internet platform," Computers \& Industrial Engineering, vol. 135, pp. 1025-1035, 2019.

[26] J. L. Wang, C. Q. Xu, J. Zhang, J. S. Bao, and R. Zhong, “A collaborative architecture of the industrial internet platform for manufacturing systems," Robotics and Computer-Integrated Manufacturing, vol. 61, Article ID 101854, 2020.

[27] M. Younan, E. H. Houssein, M. Elhoseny, and A. A. Ali, "Challenges and recommended technologies for the industrial 
internet of things: a comprehensive review," Measurement, vol. 151, Article ID 107198, 2020.

[28] M. Brettel, N. Friederichsen, M. Keller, and M. Rosenberg, "Network function virtualization," Network Function Virtualization: Concepts and Applicability in 5G Networks, vol. 12, pp. 37-65, 2017.

[29] C. Falkenreck and R. Wagner, "The internet of things-chance and challenge in industrial business relationships," Industrial Marketing Management, vol. 66, pp. 181-195, 2017.

[30] C. Metallo, R. Agrifoglio, F. Schiavone, and J. Mueller, "Understanding business model in the Internet of Things industry," Technological Forecasting and Social Change, vol. 136, pp. 298-306, 2018.

[31] M. L. Zapata, L. Berrah, and L. Tabourot, "Is a digital transformation framework enough for manufacturing smart products? The case of small and medium enterprises," Procedia Manufacturing, vol. 42, pp. 70-75, 2020.

[32] K. Y. Akdil, A. Ustundag, and E. Cevikcan, "Maturity and readiness model for industry 4.0 strategy," in Industry 4.0: Managing the Digital TransformationSpringer, Cham, Switzerland, 2018.

[33] R. S. Wadhwa, "Flexibility in manufacturing automation: a living lab case study of Norwegian metalcasting SMEs," Journal of Manufacturing Systems, vol. 31, no. 4, pp. 444-454, 2012.

[34] S. Mittal, M. A. Khan, D. Romero, and T. Wuest, "A critical review of smart manufacturing \& Industry 4.0 maturity models: implications for small and medium-sized enterprises (SMEs)," Journal of Manufacturing Systems, vol. 49, pp. 194-214, 2018.

[35] E. Müller and H. Hopf, "Competence center for the digital transformation in small and Medium-Sized enterprises," Procedia Manufacturing, vol. 11, pp. 1495-1500, 2017.

[36] E. B. Hansen and S. Bøgh, "Artificial intelligence and internet of things in small and medium-sized enterprises: a survey," Journal of Manufacturing Systems, vol. 58, pp. 362-372, 2021.

[37] R. Y. Zhong, X. Xu, E. Klotz, and S. T. Newman, "Intelligent manufacturing in the context of industry 4.0: a review," Engineering, vol. 3, no. 5, pp. 616-630, 2017.

[38] W. Li, K. Liu, M. Belitski, A. Ghobadian, and N. O’Regan, "E-leadership through strategic alignment: an empirical study of small- and medium-sized enterprises in the digital age," Journal of Information Technology, vol. 31, no. 2, pp. 185-206, 2016.

[39] W. Z. Khan, M. H. Rehman, H. M. Zangoti, M. K. Afzald, N. ArmiaM, and K. Salah, "Industrial internet of things: recent advances, enabling technologies and open challenges," Computers \& Electrical Engineering, vol. 81, Article ID 106522, 2020.

[40] O. Galor and D. N. Weil, "Population, technology, and growth: from Malthusian stagnation to the demographic transition and beyond," The American Economic Review, vol. 90, no. 4, pp. 806-828, 2000.

[41] D. Friedman, "Evolutionary games in economics," Econometrica, vol. 59, no. 3, pp. 637-666, 1991.

[42] S. Tisue and U. Wilensky, "NetLogo: design and implementation of a multi-agent modeling environment," in Proceedings of AgentSpringer, Cham, Switzerland, 2004. 